University of Wollongong

Research Online

Australian Institute for Innovative Materials -

Papers

Australian Institute for Innovative Materials

$1-1-2017$

Demetallatation of electrochemically polymerised Mn porphyrin anion / PEDOT composites under light-illumination

Danijel Boskovic

University of Wollongong, db103@uowmail.edu.au

Sivakumar Balakrishnan

CSIRO, University of Wollongong

Pawel W. Wagner

University of Wollongong, pawel@uow.edu.au

Gerhard F. Swiegers

University of Wollongong, swiegers@uow.edu.au

Follow this and additional works at: https://ro.uow.edu.au/aiimpapers

Part of the Engineering Commons, and the Physical Sciences and Mathematics Commons

Research Online is the open access institutional repository for the University of Wollongong. For further information contact the UOW Library: research-pubs@uow.edu.au 


\title{
Demetallatation of electrochemically polymerised Mn porphyrin anion / PEDOT composites under light-illumination
}

\author{
Abstract \\ This work reports photo-demetallation studies of thin-layer, electropolymerized \\ poly(3,4-ethylenedioxythiophene) (PEDOT) loaded with low levels of: (i) an anionic Mn porphyrin monomer \\ (5,10,15,20-tetrakis(4-sulfonatophenyl)porphyrinato manganese(III) chloride (MnTPPS)), and (ii) an \\ anionic Mn porphyrin polymer (poly(5-(4-vinylphenyl)-10,15,20-tris(4-sulfonatophenyl)) porphyrinato \\ manganese(III) chloride (MnPVTPPS)). UV-vis and other measurements confirm that, like previously- \\ studied cationic Mn(III) porphyrins embedded within vapour-phase polymerized PEDOT in low loadings, \\ de-metallation under light illumination also occurs in these systems. However, it occurs to a significantly \\ lesser degree. It can be concluded that demetallation appears to be an inherent feature of PEDOT \\ coatings containing low levels of Mn porphyrins when they are illuminated with light. The demetallation \\ process does not depend on the mode of polymerisation or the nature of the Mn porphyrin dopant. These \\ findings have potentially important implications in water-splitting photocatalysis by Mn porphyrin-doped \\ conducting polymers.

\section{Disciplines} \\ Engineering | Physical Sciences and Mathematics

\section{Publication Details} \\ Boskovic, D., Balakrishnan, S., Wagner, P. \& Swiegers, G. F. (2017). Demetallatation of electrochemically \\ polymerised Mn porphyrin anion / PEDOT composites under light-illumination. Synthetic Metals, 228 \\ 58-63.
}




\title{
Demetallatation of Electrochemically Polymerised Mn Porphyrin Anion I PEDOT Composites under Light-Illumination
}

\author{
Danijel Boskovic, Sivakumar Balakrishnan, Pawel Wagner, ${ }^{*}$ and Gerhard F. \\ Swiegers*
}

Intelligent Polymer Research Institute and ARC Centre of Excellence for Electromaterial Science, Innovation Campus, University of Wollongong, Wollongong, NSW 2522, Australia

Received date (to be automatically inserted after your manuscript is submitted) Accepted date (to be automatically inserted after your manuscript is accepted)

\begin{abstract}
This work reports photo-demetallation studies of thin-layer, electropolymerized, poly(3,4ethylenedioxythiophene (PEDOT) loaded with low levels of: (i) an anionic Mn porphyrin monomer (5,10,15,20-tetrakis(4-sulfonatophenyl)porphyrinato manganese(III) chloride (MnTPPS)), and (ii) an anionic Mn porphyrin polymer (poly(5-(4-vinylphenyl)-10,15,20-tris(4-sulfonatophenyl)) porphyrinato manganese(III) chloride (MnPVTPPS)). UV-visible and other measurements confirm that, like previously-studied cationic Mn(III) porphyrins embedded within vapour-phase polymerized PEDOT in low loadings, de-metallation under light illumination also occurs in these systems. However, it occurs to a significantly lesser degree. It can be concluded that demetallation appears to be an inherent feature of PEDOT coatings containing low levels of Mn porphyrins when they are illuminated with light. The demetallation process does not depend on the mode of polymerisation or the nature of the Mn porphyrin dopant. These findings have potentially important implications in water-splitting photocatalysis by $\mathrm{Mn}$ porphyrin-doped conducting polymers.
\end{abstract}

KEYWORDS: PEDOT, de-metallation, Mn porphyrin, Light-induced, photocatalysis

*Correspondence to: Pawel Wagner, email: pawel@uow.edu.au, and Gerhard F. Swiegers, email: swiegers@uow.edu.au, tel: +61-24221-3127, fax: +61-2-4221-3114 


\section{INTRODUCTION}

Several selective water-splitting photocatalysts comprising Mn porphyrins doped in conducting polymers, such as poly(terthiophene) and poly(3,4-ethylenedioxythiophene (PEDOT), have been described in the scientific literature [1(a)(b)]. In a recent publication [1(c)], we reported a novel demetallation of a Mn porphyrin in poly(3,4ethylenedioxythiophene) (PEDOT) under light illumination. A proportion of 5,10,15,20-tetraphenylporphyrinato manganese(III), Mn(III)TPP ${ }^{+}$became demetallated after it was embedded within the conducting polymer using vapourphase polymerization, leaving free and protonated ТPР (ТPP=5,10,15,20-tetraphenylporphyrin). The proportion that was demetallated declined as the loading level of the $\mathrm{Mn}(\mathrm{III}) \mathrm{TPP}^{+}$in the PEDOT was increased, consistent with the demetallation reaction being driven by light-illumination and being limited by the optical transparency of the PEDOT. However, it was not clear whether the mode of polymerisation employed, namely vapour phase polymerisation, or the cationic nature of the Mn porphyrin species, were involved in the demetallation process. The role of the PEDOT was also not clear. PEDOT has been separately reported to be capable of acting as both an electron acceptor and as an electron donor under suitable conditions [2]. In this work we report further studies seeking to clarify these questions. The preparation, characterisation, and properties of PEDOT:PSS (PSS= poly(sodium 4-styrenesulfonate)) incorporating the following have been investigated:

(i) poly(5-(4-vinylphenyl)-10,15,20-tris(4-sulfonatophenyl)) porphyrinato manganese(III) chloride (MnPVTPPS)

(Figure 1) [3], and

(ii) 5,10,15,20-tetrakis(4-sulfonatophenyl)porphyrinato manganese(III) chloride (MnTPPS) (Figure 1).

In of the above cases, polymerisation was carried out electrochemically (and not by the vapour-phase route). The concentration of the porphyrins in the PEDOT coatings were also set to low loading levels similar to that employed in the earlier work [1(c)]. High loading levels of PEDOT incorporating sulfonated MnTPPS have been studied previously without noticeable demetallation being observed [1(a)-(b)]. MnPVTPPS is similar to PSS [4-7] and therefore serves as a potentially useful counter-ion for electrochemically polymerised PEDOT [5,8].

UV-visible and other measurements confirmed that de-metallation under light illumination occured in both of the above systems, however to a significantly lesser degree than observed in the earlier study [1(c)]. It can be concluded that the demetallation reaction is not related to the use of vapour phase polymerisation or the charge on the Mn porphyrin, whether anionic or cationic. Instead, demetallation appears to be an inherent feature of PEDOT coatings containing low levels of Mn porphyrins under light-illumination.

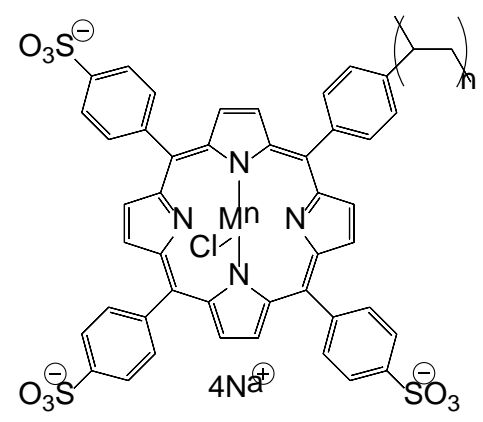

(a)

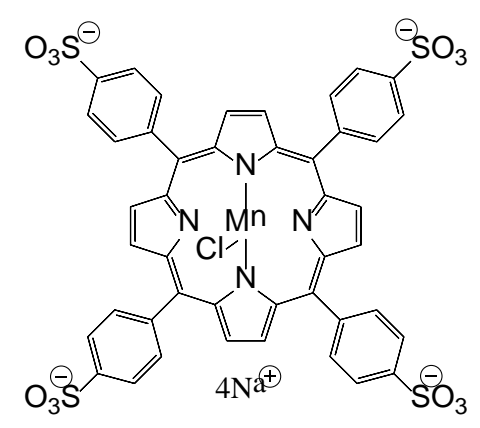

(b)

MnPVTPPS

MnTPPS

Figure 1. Schematic of (a) poly(5-(4-vinylphenyl)-10,15,20-tris(4-sulfonatophenyl)) porphyrinato manganese(III) chloride sodium salt (MnPVTPPS) and (b) its monomeric counterpart 5,10,15,20-tetrakis(4-sulfonatophenyl)porphyrinato manganese(III) chloride sodium salt (MnTPPS). 

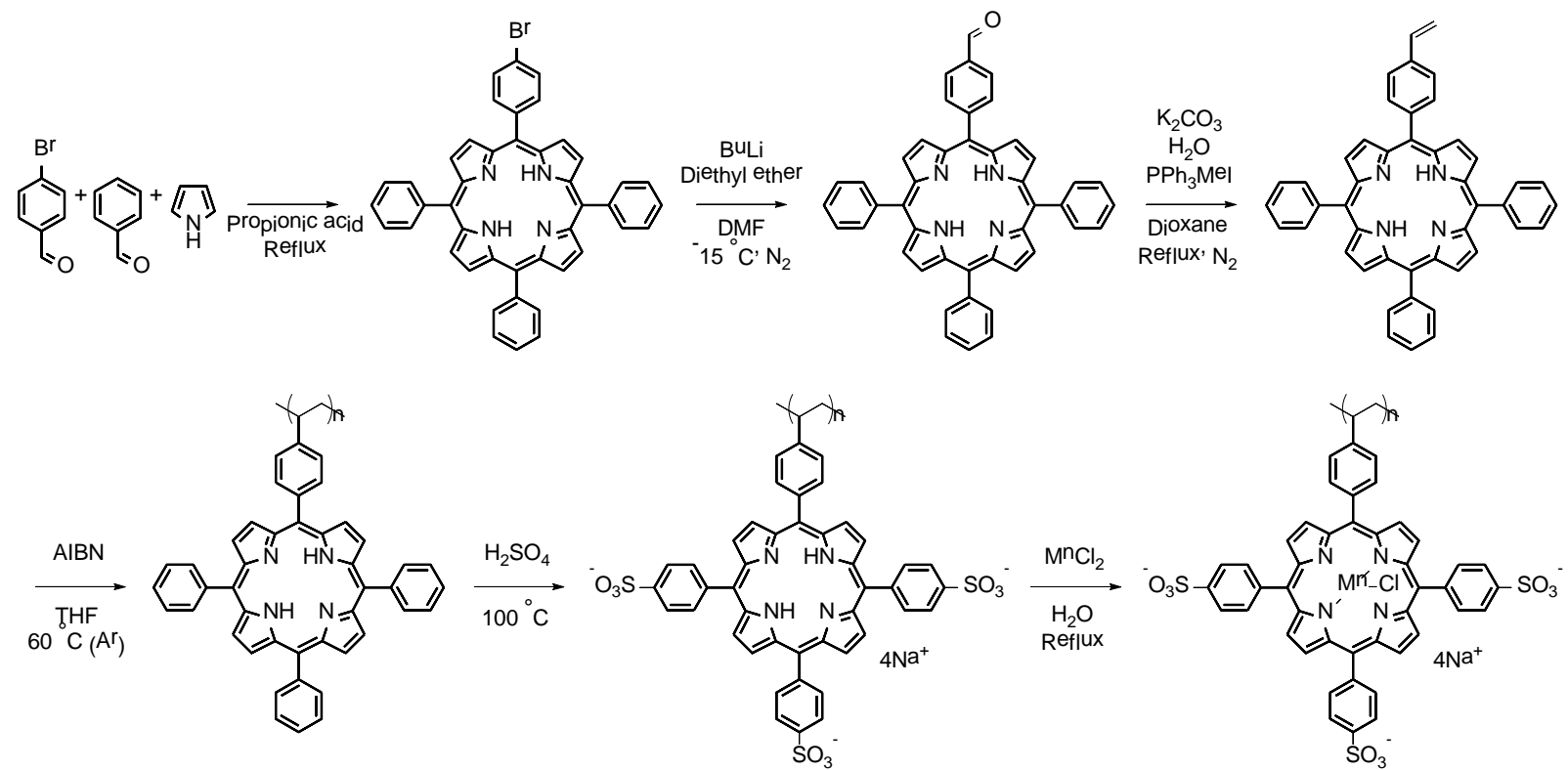

Figure 2. Synthesis of poly(5-(4-vinylphenyl)-10,15,20-tris(4-sulfonatophenyl)) porphyrinato manganese (III) chloride sodium salt) (MnPVTPPS).

\section{EXPERIMENTAL}

\subsection{Materials and methods}

The following materials were used as supplied: Fluorine doped tin oxide (FTO) slides (Zhuhai Kaivo Electronic Components Co.), glass microscope slides (Australia Optics Superstore), 3,4-ethylenedioxythiophene (EDOT) monomer, pyridine (Thermo Fisher Scientific Australia), iron(III) p-toluenesulfonate (Fe(III)-pTS) (Sigma Aldrich), 5,10,15,20-tetraphenylporphyrin (TPP) (Sigma Aldrich Co.), acetone (Sigma Chemicals), ethanol (Sigma Chemicals), and sodium sulphate $\mathrm{Na}_{2} \mathrm{SO}_{4}$ (Aim Chemicals). De-ionized water was used in all experiments. TPPS and MnTPPS were prepared following a previously published procedure [2,4].

\subsection{Synthesis of poly(5-(4-vinylphenyl)-10,15,20-tris(4-sulfonatophenyl)) porphyrinato manganese (III) chloride sodium salt (MnPVTPPS)}

The synthesis of poly(5-(4-vinylphenyl)10,15,20-tri(4-sulfonatophenyl)porphyrinato manganese (III) chloride sodium salt) (MnPVTPPS) was based on a previously described approach $[3,9,10]$. The synthesis required a number of steps, starting with the formation of 5-(4-bromophenyl)-10,15,20-triphenylporphyrin, conversion to the formyl derivative by reaction with n-butyllithium and DMF, subsequent vinyl formation by Wittig condensation with triphenylmethylphosphonium iodide, polymerisation of the vinyl group with azobisisobutyronitrile (AIBN), sulfonation, and finally manganese insertion. A detailed experimental procedure for the formation of 5-(4-bromophenyl)-10,15,20triphenylporphyrin and its conversion to 5-(4-vinylphenyl)-10,15,20-triphenyl porphyrin (VTPP) is provided in the supplementary section.

The vinyl porphyrin VTPP (0.49 g, $0.76 \mathrm{mmol})$ was dissolved in a small quantity of THF (0.7 mL) with AIBN (10-20 $\mathrm{mg}$, in $12 \%$ acetone solution), which was degassed in an ampoule following the freeze pump thaw method. The mixture was submerged in liquid nitrogen until fully frozen, then a vacuum applied for 20 min. The pump was thereafter disconnected, with the flask sealed and the mixture gently thawed in a warm water bath. This procedure was repeated 35 times after which the mixture was stirred at $60{ }^{\circ} \mathrm{C}$ under Ar atmosphere for $2.5 \mathrm{~d}$. The product was then precipitated with acetone and filtered through a sintered glass funnel under vacuum (43 mg, $0.07 \mathrm{mmol}, 9 \%$ yield). The synthesis had to be repeated several times as TLC tests showed only partial polymerisation in successive steps. The final product, 
poly(5-(4-vinylphenyl)-10,15,20-triphenylporphyrin) (PVTPP), was characterised using gel permeation chromatography (GPC) with polystyrene fractions, which indicated an average molecular weight of $47 \mathrm{kDa}$ at a fraction of $55 \%$. Smaller polymer chains at $3 \mathrm{kDa}$ and single units at $1 \mathrm{kDa}$ were found at respective fractions of $18 \%$ and $27 \%$. As the exclusion mechanism of polymers with bulky side groups may differ from that of polystyrene, these results may not be fully representative.

The polymeric porphyrin PVTPP (43 mg, $0.07 \mathrm{mmol}$ ) was then sulfonated by dissolving in concentrated $\mathrm{H}_{2} \mathrm{SO}_{4}(2$ $\mathrm{mL}$ ) and stirring at $100{ }^{\circ} \mathrm{C}$ for $4 \mathrm{~h}$, after which it was left to cool overnight. The mixture was poured into $25 \mathrm{~mL}$ of Milli-Q water and neutralised with a small quantity of $\mathrm{NaOH}$. It was purified using dialysis tubing (12-14 kDa) and Milli-Q water over several days with frequent change of water and the product poly(5-(4-vinylphenyl)-10,15,20-tris(4sulfonatophenyl)porphyrin sodium salt) (PVTPPS) obtained by evaporating the solvent and drying in a vacuum oven at $60{ }^{\circ} \mathrm{C}$ (37 mg, $0.04 \mathrm{mmol}$, 59\% yield).

In the final step PVTPPS (37 mg, $0.04 \mathrm{mmol})$ was dissolved in Milli-Q water $(20 \mathrm{~mL})$ and $\mathrm{MnCl}_{2}(0.58 \mathrm{~g}, 4.61$ mmol) added. This mixture was stirred at reflux for $25 \mathrm{~h}$ after which it was dialysed (12-14000 kDa tubing) and the solvent evaporated under vacuum, giving the desired product poly(5-(4-vinylphenyl)-10,15,20-tris(4sulfonatophenyl)porphyrinato manganese (III) chloride sodium salt) (MnPVTPPS) (56 mg, 0.05 mmol, 100\% yield). UV-Vis and MALDI analysis of the compound was in agreement with literature findings [3,9,10].

\subsection{Electrochemical polymerisation of porphyrin/PEDOT composites, MnTPPS/PEDOT:PSS and MnPVTPPS/PEDOT:PSS, on FTO glass}

A method was developed following a literature procedure [5,8] for electrochemical polymerisation (EP) of PEDOT:PSS in water. A reagent mixture was prepared containing $5 \mathrm{mM}$ of 3,4-ethylenedioxythiophene (EDOT) (0.71 $\mathrm{mg} / \mathrm{ml}$ ) and $2.5 \mathrm{mM}$ PSS (by monomer mass $0.46 \mathrm{mg} / \mathrm{mL}$ ) in water. The EDOT solution was prepared, first, in $10 \mathrm{~mL}$ volume, carefully sonicated and vortex-stirred until completely dissolved. Approximately $2 \mathrm{~mL}$ were transferred to a second sample vial containing the PSS. The solution was again sonicated and mixed to ensure proper dissolution of the reagents.

For the samples containing porphyrins, the relevant amount of Mn porphyrin (either MnTPPS or MnPVTPPS) was weighed out before and the EDOT/PSS mixture added to dissolve into it. The porphyrins MnTPPS $(2.5 \mathrm{mg} / \mathrm{ml}, 2.25$ $\mathrm{mM}$ ) or MnPVTPPS (0.86 mg/mL, $0.83 \mathrm{mM}$ ) were included in separate EDOT/PSS mixtures.

An FTO glass electrode was sonicated in acetone, rinsed with water and allowed to dry before fitting a copper cable with silver paste and epoxy resin to it. Prior to polymerisation, the electrode was plasma-cleaned for $15 \mathrm{~min}$.

The above samples were electropolymerised in a small vessel, into which the reagent mixture ( $\sim .9 \mathrm{~mL}$ ) was injected. A BASi miniature $\mathrm{Ag} / \mathrm{AgCl}$ reference electrode was inserted. The electrodes were connected to an EDAQ466 potentiostat and cyclic voltammetry (CV) was performed on the sample at $-0.4-+1.2 \mathrm{~V}$ (vs Ag/AgCl) for 50 cycles at $50 \mathrm{mV} / \mathrm{s}$. The sample was submerged in water to wash out impurities, and then left to air dry. Samples were prepared in duplicate. The finished film was analysed by UV-Vis spectroscopy and electrochemistry.

\section{$2.4 \quad$ Electrochemical Testing}

All electrochemical measurements were carried out in $0.1 \mathrm{M} \mathrm{Na}_{2} \mathrm{SO}_{4}$ on an EDAQ466 potentiostat. Linear sweep voltammograms were performed at a scan rate of $5 \mathrm{mV} / \mathrm{s}$. The electrochemical cell employed comprised of a rectangular chamber with fixed positions for the working electrode, reference electrode $(\mathrm{Ag} / \mathrm{AgCl})$ and counter electrode $(\mathrm{Pt}$ mesh; ca. $1.0 \times 1.5 \mathrm{~cm}$ ). The distance between the working and counter-electrode was $25 \mathrm{~mm}$.

The PEDOT and Mn porphyrin/PEDOT samples were subjected to electrochemical testing in a cell filled with $\mathrm{Na}_{2} \mathrm{SO}_{4}(0.1 \mathrm{M})$ that had been degassed with $\mathrm{N}_{2}$ gas while stirring for $1 \mathrm{~h}$. A SoLux daylight MR16 halogen light bulb 
(12 V, $50 \mathrm{~W}, 24^{\circ}$; ca. 0.25 sun intensity) was used with a stable output range of 275 - $750 \mathrm{~nm}$. The lamp only approximated sunlight conditions. The light was passed through a $3 \mathrm{~mm}$ glass filter to remove the infra-red component, before reaching the sample. The cut-off filter blocked all wavelengths above $700 \mathrm{~nm}$.

Before testing, cyclic voltammetry (CV) was performed on the film for 20 cycles at $10 \mathrm{mV} / \mathrm{sec}$ in order to stabilise the film. LSV sweeps were then performed over the range of $0-0.7 \mathrm{~V}$ at a scan rate of $5 \mathrm{mV} / \mathrm{sec}$. Voltages beyond $0.7 \mathrm{~V}$ (vs $\mathrm{Ag} / \mathrm{AgCl}$ ) were not studied in order to avoid oxidative damage to the film. In a typical experiment, five sweeps were done in the dark and then three sweeps with light illumination turned on. This ensured that the LSV spectrum remained constant over the course of the data recording as it was otherwise affected by charge imbalances brought on by static electricity. The last of the LSV sweeps with and without illumination were used as data. This method was applied to all of the films investigated. In all cases the resulting current (in $\mu \mathrm{A}$ ) was converted into current density (in $\mu \mathrm{A} / \mathrm{cm}^{2}$ ) by dividing by the geometric area of the film.

Following the LSV measurement, each sample was tested by CA (chronoamperometry) at the potential of $0.65 \mathrm{~V}$ ( $v \mathrm{~s}$ $\mathrm{Ag} / \mathrm{AgCl})$. In a typical experiment the chosen voltage was applied to the film for $1 \mathrm{~h}$ without illumination to equilibrate the baseline current. The light was then switched on to measure the resulting photocurrent for 10 min and then turned off. The data provided in the section below were taken from 1 min before illumination to 1 min after the end of illumination. The data (in $\mu \mathrm{A}$ ) was normalised by subtracting the baseline before illumination and the results converted to current density (in $\mu \mathrm{A} / \mathrm{cm}^{2}$ ).

\section{RESULTS AND DISCUSSION}

\subsection{Electropolymerisation of PEDOT:PSS and porphyrin/PEDOT:PSS}

Electropolymerisation of PEDOT:PSS on FTO glass was carried out as described in the experimental section [7]. In the first run, control PEDOT:PSS was synthesised. Thereafter MnTPPS or MnPVTPPS (as co-mixtures with PSS) were incorporated at low concentrations similar to our previous report [1(c)], with MnTPPS incorporated at $2.5 \mathrm{mg} / \mathrm{ml}$ and MnPVTPPS at $0.86 \mathrm{mg} / \mathrm{mL}$. Under these circumstances the molar ratio of the MnTPPS in PEDOT was previously determined to be ca. 60 PEDOT repeat units : 1 porphyrin. Because of the multi-step synthesis required, only a small quantity of VTPPS was prepared and only one concentration of MnPVTPPS in PEDOT studied. Due to a paucity of material, it was not possible to accurately determine its molar ratio of PEDOT : porphyrin.

The initial trial of PEDOT:PSS, without porphyrin, under the conditions of the present study, exhibited a CV spectrum that was characteristic of gradually increasing capacitive current, indicating a progressive build-up of conducting polymer on the electrode (Figure S1). The porphyrins MnTPPS or MnPVTPPS were later separately added (both with PSS), generating the materials PEDOT:PSS, MnTPPS/PEDOT:PSS and MnPVTPPS/PEDOT:PSS. The films were either deep blue (PEDOT:PSS, Figure S2(a)) or deep green (MnTPPS/PEDOT:PSS; Figure S2(b) and MnPVTPPS/PEDOT:PSS Figure S2(c)).

The electropolymerised (EP) PEDOT:PSS film was tested by Linear Sweep Voltammetry (LSV) and Chronoamperometry (CA) under light illumination (Figure 3). The LSV spectrum of the control, porphyrin-free PEDOT:PSS film featured a weak photocurrent with onset potential ca. $0.5 \mathrm{~V}$ (vs Ag/AgCl). This was similar to that previously observed for vapour phase polymerised (VPP) PEDOT [1(c)]. However, while vapour phase polymerised VPP PEDOT typically displayed a photocurrent of ca. $1 \mu \mathrm{A} / \mathrm{cm}^{2}$ at $0.65 \mathrm{~V}$ in LSVs, the electropolymerised EP PEDOT:PSS exhibited photocurrent densities up to as high as $8 \mu \mathrm{A} / \mathrm{cm}^{2}$. This indicated that the EP PEDOT:PSS was more light active, even without a porphyrin species present. 


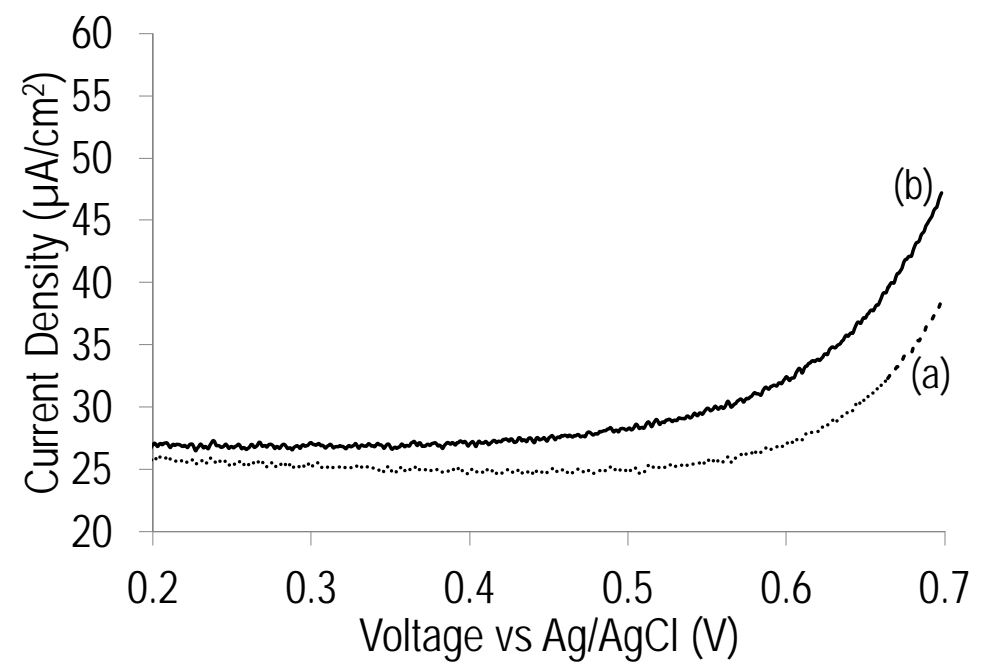

Figure 3. LSV spectra of PEDOT:PSS (without MnTPPS), (a) in the dark, (b) with light illumination.

\subsection{Photoelectrochemical testing of porphyrin-free, control PEDOT:PSS}

The CA photocurrent density measurement of PEDOT:PSS reflected the increased photocurrents observed in the LSV experiment, reaching a level of $3.89 \mu \mathrm{A} / \mathrm{cm}^{2}$ after $10 \mathrm{~min}$ of illumination at $0.65 \mathrm{~V}$ (Figure 4). This was significantly greater than the $0.75 \mu \mathrm{A} / \mathrm{cm}^{2}$ previously measured for VPP PEDOT [1(c)].

\subsection{Photoelectrochemical testing of MnTPPS/PEDOT:PSS}

The EP MnTPPS/PEDOT:PSS film was tested by LSV and CA to measure the photocurrent produced under illumination. The LSV spectrum of MnTPPS/PEDOT:PSS was similar to that of PEDOT:PSS, also displaying an onset potential from ca. $0.5 \mathrm{~V}$ (vs Ag/AgCl) (Figure 5). In this case however, the photocurrents were very similar to that of vapour-phase polymerized MnTPPS/PEDOT reported previously where the onset was ca. $0.55 \mathrm{~V}$ (vs. Ag/AgCl) [1(c)].

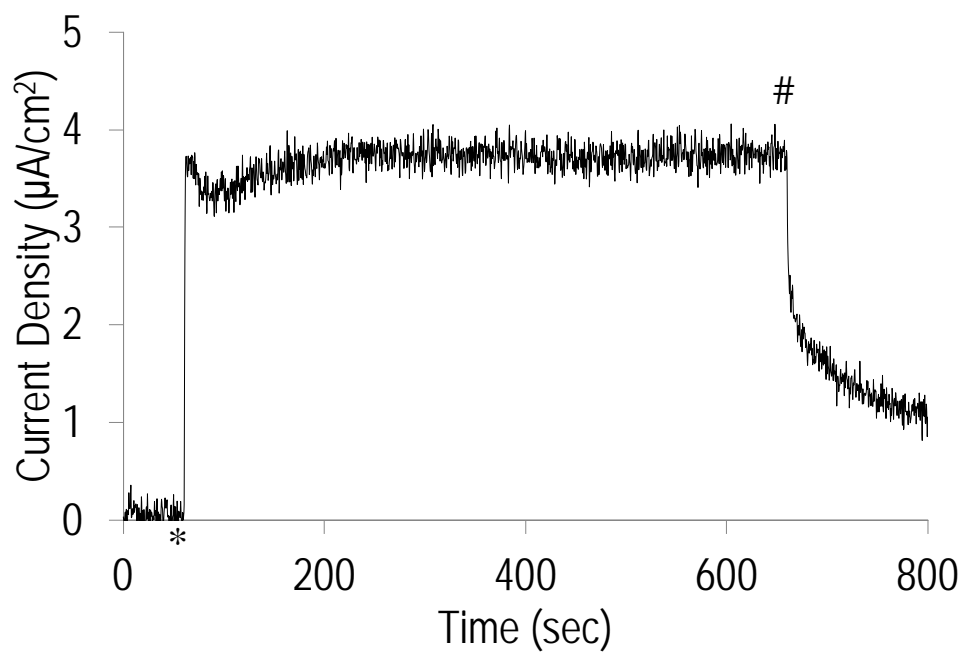

Figure 4. Chronoamperogram under light illumination at $0.65 \mathrm{~V}$ (vs Ag/AgCl) of PEDOT:PSS. (* = "light on"; \# = "light off") 


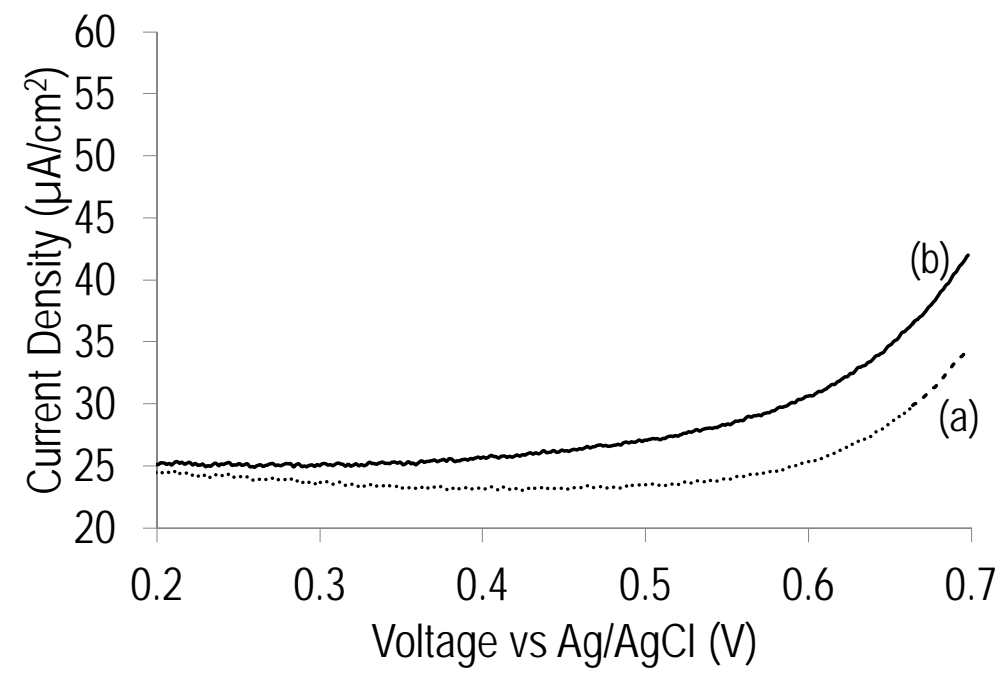

Figure 5. LSV spectra of MnTPPS/PEDOT:PSS, (a) in the dark, (b) with light illumination.

The CA photocurrent density measurement for MnTPPS/PEDOT:PSS was $3.29 \mu \mathrm{A} / \mathrm{cm}^{2}$ at $0.65 \mathrm{~V}$ (vs Ag/AgCl) (Figure 6), which was, in fact, marginally lower than that of PEDOT:PSS (Table 1), but higher than that of the highest performing vapour-phase polymerized MnTPPS/PEDOT (at $1.46 \mu \mathrm{A} / \mathrm{cm}^{2}$ ) [1(c)].

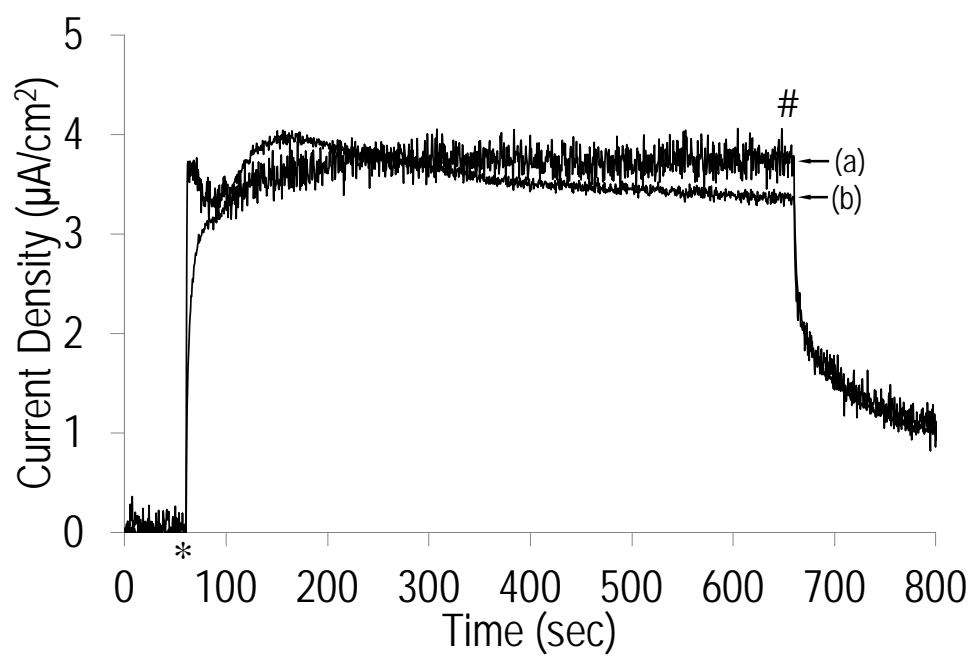

Figure 6. Chronoamperograms under light illumination at $0.65 \mathrm{~V}$ (vs Ag/AgCl) for (a) PEDOT:PSS and (b) MnTPPS/PEDOT:PSS (* = "light on"; \# = "light off")

\begin{tabular}{|c|c|}
\hline Sample & $\begin{array}{c}\text { Current Density at } \mathbf{0 . 6 5} \mathbf{~ V} \\
\text { under light illumination } \\
\left(\boldsymbol{\mu} \mathbf{A} / \mathbf{c m}^{2}\right)\end{array}$ \\
\hline MnTPPS/PEDOT:PSS & 3.29 \\
\hline PEDOT:PSS & 3.89 \\
\hline
\end{tabular}

Table 1. Photocurrent density of MnTPPS/PEDOT:PSS at $0.65 \mathrm{~V}$ (vs Ag/AgCl). 

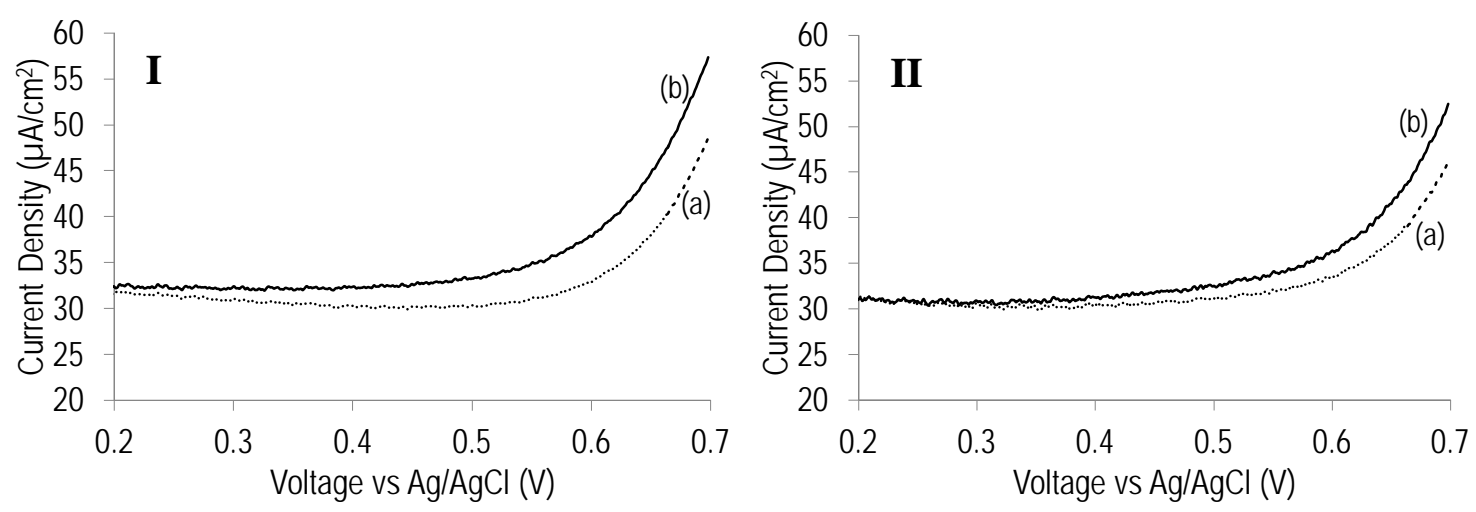

Figure 7. LSV spectra of (I) MnPVTPPS/PEDOT:PSS 1 and (II) MnPVTPPS/PEDOT:PSS 2, (a) in the dark, (b) with light illumination.

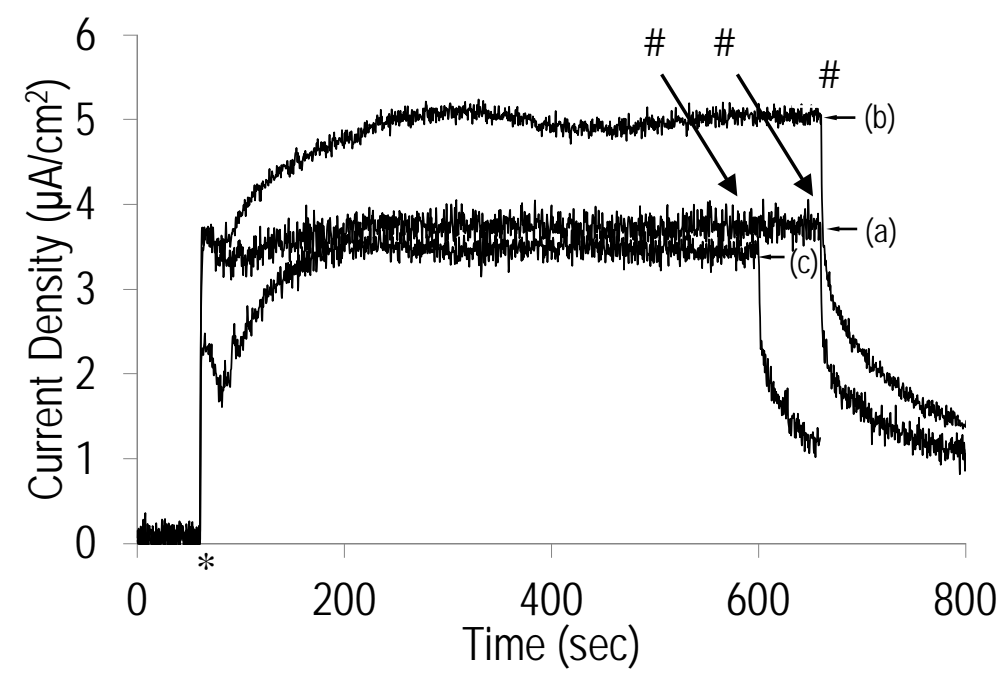

Figure 8. Chronoamperogram under light illumination at $0.65 \mathrm{~V}$ (vs $\mathrm{Ag} / \mathrm{AgCl}$ ) of (a) control PEDOT:PSS, (b) MnPVTPPS/PEDOT:PSS 1 and (c) MnPVTPPS/PEDOT:PSS 2. (* = "light on"; \# = "light off")

\begin{tabular}{|c|c|}
\hline Sample & $\begin{array}{c}\text { Current Density at } \mathbf{0 . 6 5} \mathbf{~ V} \\
\left(\mathbf{\mu} \mathbf{A} / \mathbf{c m}^{2}\right)\end{array}$ \\
\hline MnPVTPPS/PEDOT:PSS 1 & 5.00 \\
\hline MnPVTPPS/PEDOT:PSS 2 & 3.39 \\
\hline PEDOT:PSS & 3.89 \\
\hline
\end{tabular}

Table 2. Photocurrent density of MnPVTPPS/PEDOT:PSS under light illumination at $0.65 \mathrm{~V}$ (vs Ag/AgCl). 


\section{4}

\section{Photoelectrochemical testing of MnPVTPPS/PEDOT:PSS}

The EP MnPVTPPS/PEDOT:PSS film was tested by LSV and CA to ascertain the photocurrent produced under light illumination. The LSV spectra of MnPVTPPS/PEDOT:PSS exhibited, in all cases, a photocurrent onset potential of ca. $0.5 \mathrm{~V}$ (vs Ag/AgCl) (Figure 7).

During the CA testing of multiple MnPVTPPS/PEDOT:PSS samples, two distinctly different photocurrent profiles were detected. Each sample tested fell into one of these profiles, which have been distinguished here as MnPVTPPS/PEDOT:PSS 1 and MnPVTPPS/PEDOT:PSS 2.

One set of samples (MnPVTPPS/PEDOT:PSS 1) displayed a notably larger photocurrent overall than the other set of samples (MnPVTPPS/PEDOT:PSS 2).

When tested by $\mathrm{CA}$ at $0.65 \mathrm{~V}$ (vs $\mathrm{Ag} / \mathrm{AgCl}$ ) with illumination, samples MnPVTPPS/PEDOT:PSS 1 displayed the highest photocurrent density measured so far for this class of material at $5.00 \mu \mathrm{A} / \mathrm{cm}^{2}$. Interestingly, the duplicate samples MnPVTPPS/PEDOT:PSS 2 made using, apparently, exactly the same method yielded only $3.39 \mu \mathrm{A} / \mathrm{cm}^{2}$, lower than that of PEDOT:PSS (Figure 8, Table 2). Despite strenuous attempts to identify physical factors that were different in the preparative procedures for the MnPVTPPS/PEDOT:PSS 1 and MnPVTPPS/PEDOT:PSS 2 samples, no clear distinction in this respect could be determined. No such phenomenon was observed when testing the MnTPPS/PEDOT:PSS samples.

\subsection{UV-Vis analysis of PEDOT:PSS}

The EP PEDOT:PSS film was analysed using UV-Vis spectroscopy after fabrication. It exhibited the characteristic spectrum of PEDOT in oxidised form (Figure S3); this had also been observed before in VPP PEDOT samples [8,11].

\subsection{UV-Vis analysis of MnTPPS/PEDOT:PSS}

The electropolymerised MnTPPS/PEDOT:PSS film was also analysed by UV-Vis spectroscopy. The UV-Vis spectrum of MnTPPS/PEDOT:PSS yielded absorbance peaks at $425 \mathrm{~nm}, 443 \mathrm{~nm}$ and $489 \mathrm{~nm}$ (Figure 9). These were consistent with the previously prepared VPP MnTPPS/PEDOT [1(c)], with the exception that the $489 \mathrm{~nm}$ absorbance peak fell at ca. $475 \mathrm{~nm}$ in that case. The absorbance peaks correspond to the Soret Band of the porphyrin in its initial Mn(III)TPPS form (489 nm) as well as the free $\mathrm{H}_{2}$ TPPS (425 nm) and a very small shoulder (443 nm) likely corresponding to the protonated species $\mathrm{H}_{4} \mathrm{TPPS}^{2+}$ [12].

Compared to the spectrum of MnTPPS in water, which featured the Mn(III)TPPS Soret Band at $466 \mathrm{~nm}$, the absorbance peak for the MnTPPS/PEDOT:PSS was significantly red shifted to $489 \mathrm{~nm}$, whereas the Soret band of $\mathrm{H}_{4} \mathrm{TPPS}^{2+}$ according to the scientific literature [12] aligned perfectly with the small shoulder at $443 \mathrm{~nm}$. The unusually large peak at $705 \mathrm{~nm}$, with associated shoulders at $575 \mathrm{~nm}, 625 \mathrm{~nm}$, and $675 \mathrm{~nm}$, was almost certainly due to $\mathrm{H}_{4} \mathrm{TPPS}^{2+}$ [12], also significantly red shifted. As noted previously [1,12], fully protonated porphyrins of this type typically display poorly resolved Q-band spectra, with the peak at longest wavelength significantly amplified relative to the other Q-band peaks.

Surprisingly some demetallation, with accompanying protonation of the resulting free porphyrin appeared to have also occurred in this system under electropolymerisation conditions. In our previous work, demetallation of certain cationic Mn porphyrins in vapour-phase polymerized PEDOT was observed under light illumination [1(c)]. However, the demetallation in the present case was substantially less than that oberved in the previous study. It does demonstrate however, that the demetallation process is independent of the method of polymerisation. 


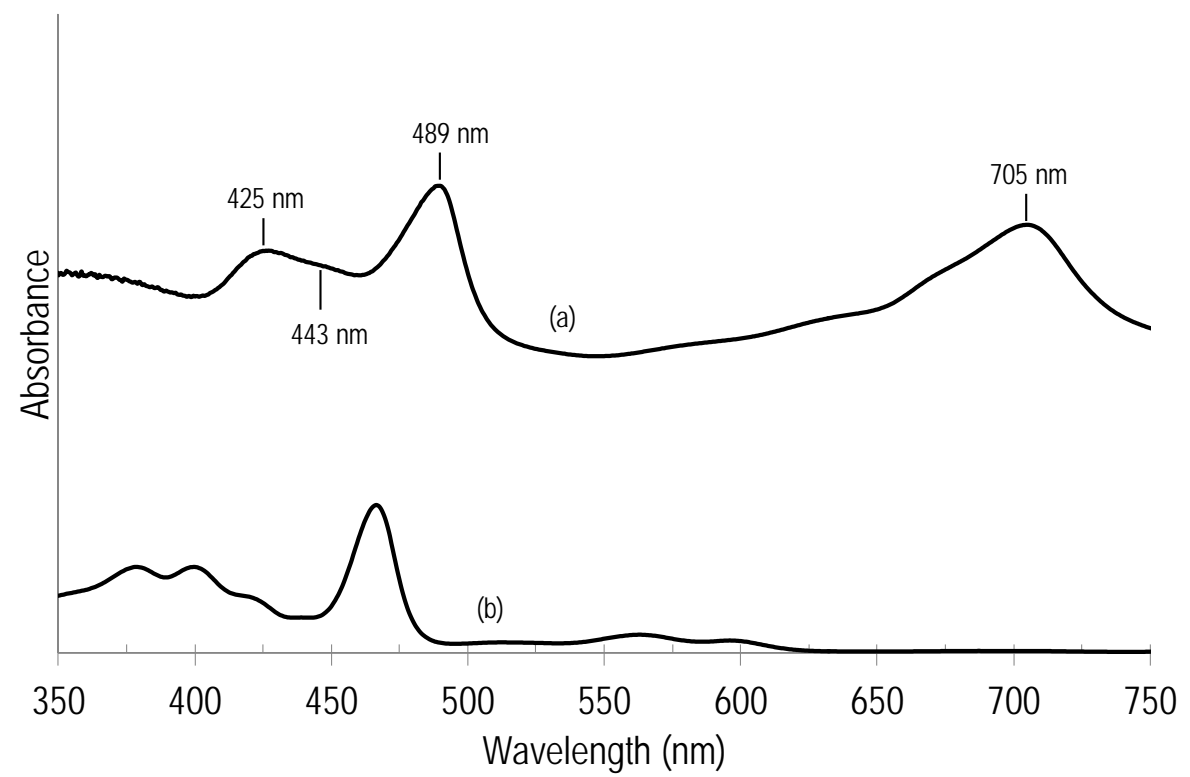

Figure 9. UV-Vis spectrum of (a) PEDOT:PSS:MnTPPS and (b) MnTPPS in water.

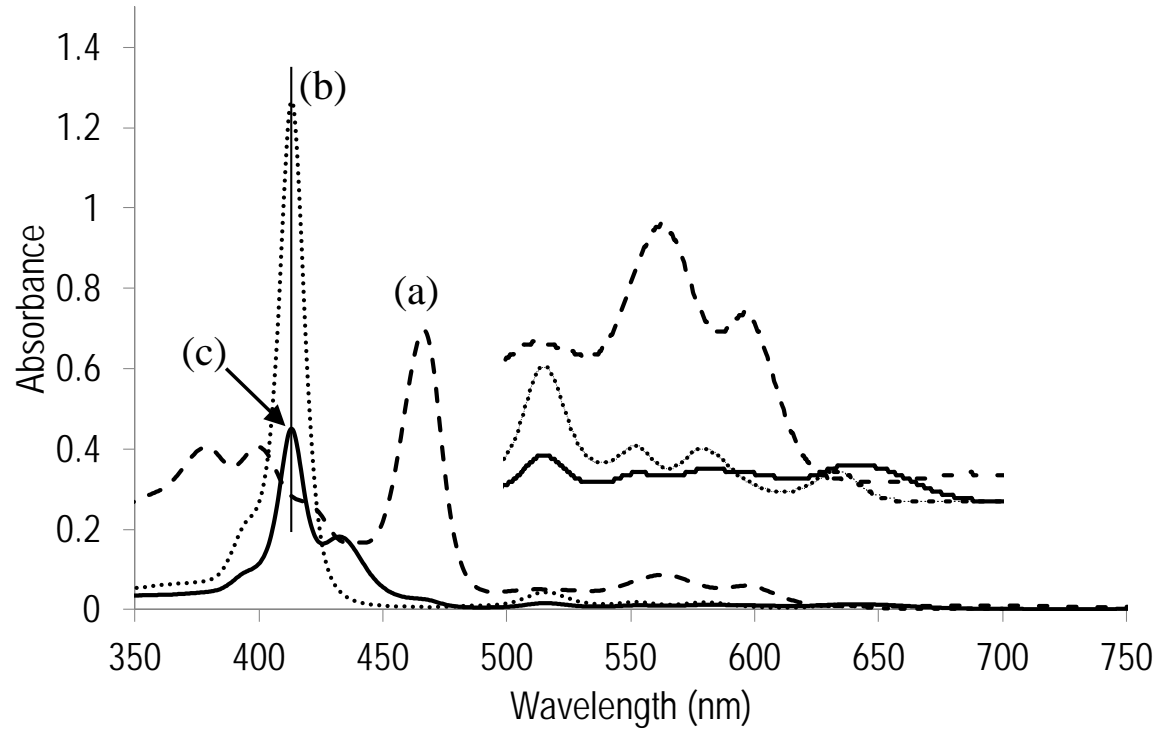

Figure 10. UV-Vis spectra in water of: (a) MnTPPS, (b) TPPS, and (c) the water rinse from MnTPPS/PEDOT:PSS after fabrication.

An analysis was conducted on the water rinsed off the MnTPPS/PEDOT:PSS film after electrodeposition and the UV-Vis spectrum of the eluate was compared to the spectra of MnTPPS and TPPS in water solution (Figure 10). The comparison showed that the porphyrin species washed out of the film after polymerisation was indeed the free TPPS with a Soret Band peak at ca. 410 nm (Figure 10(b),(c)), rather than MnTPPS (Figure 10(a),(c))).

A small portion of what was likely protonated TPPS was also identified in a band at ca. $440 \mathrm{~nm}$ along with an amplified Q-band peak at ca. $650 \mathrm{~nm}$. However it should be noted that these washings represented only the loosely bound species that adhered to the film in excess after polymerisation, and were not characteristic of the entirety of the porphyrins in the sample. The previous UV-Vis spectrum of the MnTPPS/PEDOT:PSS film (Figure 9) had 
demonstrated unequivocally that large proportions of MnTPPS were still present in the film as evidenced by the significant absorbance peak corresponding to Mn(III)TPPS at $489 \mathrm{~nm}$.

\subsection{UV-Vis analysis of MnPVTPPS/PEDOT:PSS 1 and MnPVTPPS/PEDOT:PSS 2}

The samples containing the polymeric porphyrin MnPVTPPS was of great interest as one form of them displayed the highest photocurrent so far recorded in studies of Mn porphyrin PEDOT films. As noted above, despite being made by an identical procedure using the same sample of MnPVTPPS, two distinctly different types of composites were observed - MnPVTPPS/PEDOT:PSS 1 and MnPVTPPS/PEDOT:PSS 2. The former yielded relatively high photocurrents, while the latter yielded photocurrents that were identical to the control PEDOT:PSS. To try to explain the difference in photocurrent performance for MnPVTPPS/PEDOT:PSS 1 and MnPVTPPS/PEDOT:PSS 2, UV-Vis absorbance analysis was carried out.

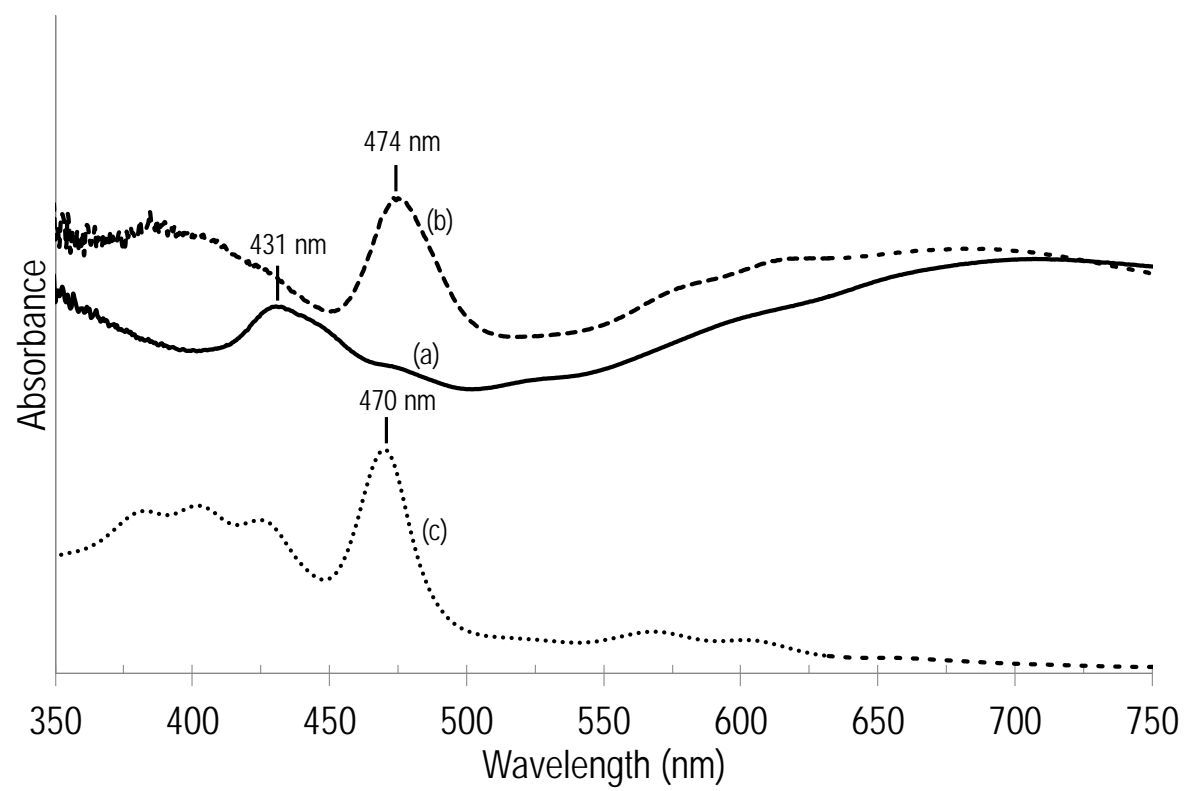

Figure 11. Representative UV-Vis spectra of (a) MnPVTPPS/PEDOT:PSS 1, (b) MnPVTPPS/PEDOT:PSS 2 and (c) MnPVTPPS in water.

The UV-Vis spectra of MnPVTPPS/PEDOT:PSS 1 and MnPVTPPS/PEDOT:PSS 2 were compared to that of MnPVTPPS in water solution (Figure 11). The high performing material, MnPVTPPS/PEDOT:PSS 1, typically displayed a dominant absorption peak at $431 \mathrm{~nm}$ with a small shoulder at $444 \mathrm{~nm}$ and a minor peak at $474 \mathrm{~nm}$. These could be attributed to the free base porphyrin $\mathrm{H}_{2}$ PVTPPS (431 nm), its acidified form (444 nm), and Mn(III)PVTPPS (474 nm) based on the similar Soret Bands observed in the $\mathrm{H}_{2}$ TPPS- $\mathrm{H}_{4} \mathrm{TPPS}$ system. As can be seen in Figure 11(a), the higher photocurrent of MnPVTPPS/PEDOT:PSS 1 appears to be associated with demetallated porphyrins and a Mn-free coating.

By contrast, the low performing sample, MnPVTPPS/PEDOT:PSS 2, exhibited only one clear absorbance peak in the Soret Band region, at $474 \mathrm{~nm}$. This indicated that the porphyrin in that sample had not been converted to the metal-free porphyrin form. That is, as can be seen in Figure 11(b), the lower photocurrent of MnPVTPPS/PEDOT:PSS 2 appears to be associated with a predominance of metallated porphyrins and the presence of $\mathrm{Mn}$ in the coating. The fact that the photocurrent of MnPVTPPS/PEDOT:PSS 2 was the same or lower than that of PEDOT:PSS suggests that the observed 
photocurrent may have been due to the PEDOT:PSS alone, with the porphyrin in MnPVTPPS/PEDOT:PSS 2 being catalytically inactive.

The photocurrent density measured for MnPVTTPS/PEDOT:PSS was the highest so far recorded for Mn porphyrin/PEDOT systems in our studies. At $0.65 \mathrm{~V}$ ( $v$ s $\mathrm{Ag} / \mathrm{AgCl}$ ) the photocurrent density for MnPVTPPS/PEDOT:PSS was $5.00 \mu \mathrm{A} / \mathrm{cm}^{2}$ compared to the highest reading of MnTPP/PEDOT at $2.91 \mu \mathrm{A} / \mathrm{cm}^{2}$ at the same voltage [1(c)].

The samples were examined by UV-Vis and demetallation was found to also occur when film fabrication was carried out using electrochemical polymerisation.

\section{CONCLUSIONS AND SUMMARY}

The phenomenon of demetallation of Mn porphyrins at low concentrations and its connection to unusually high photocurrents was previously observed in vapour-phase polymerised Mn porphyrin/PEDOT composites [1(c)]. Demetallation was hypothesized, in that case, to be a result of the cationic nature of the Mn porphyrins used in that study, the vapour phase polymerisation process employed, and the the specific conditions of light illumination thereafter. The above work confirms however, that demetallation is not associated with vapour phase polymerisation; it occurs even when electropolymerisation is used. Nor is it due to the nature of the charge on the Mn porphyrins, whether cationic or anionic since MnTPPS also becomes demetallated in PEDOT:PSS under suitably low loading conditions.

Moreover, the phenomenon of smaller photocurrents associated with the presence of the Mn(III) species was also observed in the MnPVTPPS/PEDOT:PSS. What makes these results remarkable is that the overall concentration of porphyrin present in the MnPVTPPS/PEDOT:PSS polymer coatings was necessarily constant during fabrication. That is, whereas demetallation was observed to be dependent on the loading level in vapour phase polymerised MnTPP/PEDOT, in the MnPVTPPS/PEDOT:PSS coatings there seems to have been two, concentration-independent pathways, one of which led, almost exclusively, to demetallation and the other, almost exclusively, to Mn retention. The trigger that led to one pathway over the other remains unclear. What was clear however was that the photocurrent generated depended on whether the porphyrin in the sample was in its Mn porphyrin or free base form. In agreement with our previous work [1(c)], it can be concluded that, at low loading levls, the free base TPPS generated in the PEDOT undergoes photoelectrolytic degradation creating a photocurrent in the system.

In the present work we therefore showed that demetallation also occurs, albeit less severely, with anionic Mn porphyrin loaded into electropolymerised PEDOT. It does not derive from the method of polymerisation used, nor is it associated with the charge on the Mn porphyrin, whether anionic or cationic. Rather, the demetallation reaction is independent of the method of polymerization and the Mn porphyrin dopant employed. Indeed, it appears to be an inherent feature of PEDOT coatings containing low levels of Mn porphyrins when they are illuminated with light. These findings have potentially important implications in water-splitting photocatalysis by Mn porphyrin-doped conducting polymers.

\section{Acknowledgements}

DB acknowledges financial support during his PhD by the Australian Research Council. 


\section{REFERENCES}

1. (a) Chen J, Wagner P, Tong L, Boskovic D, Zhang W, Officer D, Wallace GG, Swiegers GF Chem. Sci. 2013; 4: 2797, and references therein ; (b) Chen J, Wagner P, Swiegers GF, Officer DL, Wallace GG Angew. Chem. Int. Ed. Engl. 2012; 124: 1943 ; (c) Boskovic D, Balakrishnan S, Chen J, Wagner P, Swiegers GF J. Porph. Phthal. 2016; in press (DOI: 10.1142/S1088424616501285);

2. (a) Li X, Lu W, Dong W, Wu D. Zhou D, Chen L Nanoscale, 2013; 5: 5257. PEDOT has also been used to oxidise halides in aqueous solutions; see: Mubeen S, Lee J, Singh N, Moskovits M, McFarland EW Energy Environ. Sci., 2013; 6: 1633; (b) Winther-Jensen B, Fraser K, Ong C, Forsyth M, MacFarlane DR Adv. Mater., 2010; 22: 1727. See also: Gu C, Norris BC, Fan FRF, Bielawski W, Bard AJ ACS Catal., 2012; 2: 746.

3. Sumi K, Kimura M, Nakamura I J. Polym. Sci. A Poly. Chem. 1994; 32(7): 1243-1254.

4. Elschner A, Lövenich W, Merker U, Reuter K, PEDOT: Principles and Applications of an Intrinsically Conductive Polymer, CRC Press, Boca Raton: 2011. Harriman A, Porter G J. Chem. Soc., Faraday Trans. 2 1979; 75: 15321542.

5. Groenendaal LB, Zotti G, Aubert P.-H., Waybright SM, Reynolds JR. Adv. Mater. 2003; 15(11): 855-879; Groenendaal LB, Jonas F, Freitag D, Pielartzik H, Reynolds JR Adv. Mater. 2000; 12(7): 481-494

6. Jonas F, Morrison JT Synth. Met. 1997; 85(1-3): 1397-1398.

7. Lerch K, Jonas F, Linke M J. Chim. Phys. 1998; 95(6): 1506-1509.

8. Asami R, Atobe M, Fuchigami T J. Am. Chem. Soc. 2005; 127(38): 13160-13161.

9. Kajiwara A, Aramata K, Kamachi M Polym J 1994; 26(2): 215-223.

10. Pomogailo AD, Razumov VF, Voloshanovskii IS J. Porph. Phthalocyan. 2000; 4(1): 45-64.

11. Winther-Jensen B, West K Macromolecules 2004; 37(12): 4538-4543.

12. (a) Jimenez HR, Julve M, Faus J J. Chem. Soc. Dalton Trans. 1991; (8): 1945-1949; (b) Ramirez-Gutierrez O, Claret J, Ribo J. M. J. Porph. Phthal. 2005; 9: 436-443; (c) Dong Z, Scammells PJ J. Org. Chem. 2007, 72, 98819885. 